\title{
Approximate Near Neighbors for General Symmetric Norms*
}

\author{
Alexandr Andoni \\ Columbia University \\ New York, NY, USA 10027 \\ andoni@cs.columbia.edu
}

\author{
Huy L. Nguyen \\ Northeastern University \\ Boston, MA, USA 02115 \\ hu.nguyen@northeastern.edu
}

\author{
Aleksandar Nikolov \\ University of Toronto \\ Toronto, ON, Canada \\ anikolov@cs.toronto.edu
}

\author{
Ilya Razenshteyn \\ MIT CSAIL \\ Cambridge, MA, USA 02139 \\ ilyaraz@mit.edu
}

\author{
Erik Waingarten \\ Columbia University \\ New York, NY, USA 10027 \\ eaw@cs.columbia.edu
}

\begin{abstract}
We show that every symmetric normed space admits an efficient nearest neighbor search data structure with doubly-logarithmic approximation. Specifically, for every $n, d=n^{o(1)}$, and every $d$ dimensional symmetric norm $\|\cdot\|$, there exists a data structure for poly $(\log \log n)$-approximate nearest neighbor search over $\|\cdot\|$ for $n$-point datasets achieving $n^{o(1)}$ query time and $n^{1+o(1)}$ space. The main technical ingredient of the algorithm is a low-distortion embedding of a symmetric norm into a low-dimensional iterated product of top- $k$ norms.

We also show that our techniques cannot be extended to general norms.
\end{abstract}

\section{CCS CONCEPTS}

- Theory of computation $\rightarrow$ Nearest neighbor algorithms;

\section{KEYWORDS}

approximate near-neighbor, symmetric norms

\section{ACM Reference format:}

Alexandr Andoni, Huy L. Nguyen, Aleksandar Nikolov, Ilya Razenshteyn, and Erik Waingarten. 2017. Approximate Near Neighbors for General Symmetric Norms. In Proceedings of 49th Annual ACM SIGACT Symposium on the Theory of Computing, Montreal, Canada, fune 2017 (STOC'17), 12 pages. DOI: $10.1145 / 3055399.3055418$

\section{INTRODUCTION}

The Approximate Near Neighbor problem (ANN) is defined as follows. The input is a dataset $P$ lying in a metric space $\left(X, d_{X}\right)$, a distance threshold $r>0$, and a desired approximation $c>1$. The goal is to preprocess $P$ so that, given a query point $q \in X$, with the promise that at least one of the data points is within distance $r$, output a data point within distance $c r$ from $q$. The ANN problem is an important tool in modern data analysis, and, at the same time,

*The full version of the paper is available at [10].

Permission to make digital or hard copies of all or part of this work for personal or classroom use is granted without fee provided that copies are not made or distributed for profit or commercial advantage and that copies bear this notice and the full citation on the first page. Copyrights for components of this work owned by others than ACM must be honored. Abstracting with credit is permitted. To copy otherwise, or republish, to post on servers or to redistribute to lists, requires prior specific permission and/or a fee. Request permissions from permissions@acm.org.

STOC'17, Montreal, Canada

(c) 2017 ACM. 978-1-4503-4528-6/17/06 ..\$15.00

DOI: $10.1145 / 3055399.3055418$ is a source of many exciting theoretical developments, see, e.g., the survey in [3].

In many applications, the metric is defined on $d$-dimensional real vectors $\mathbb{R}^{d}$. Depending on the relation between the dimension $d$ and the number of data points $n$, two main regimes have emerged: lowand high-dimensional. The low-dimensional regime corresponds to $d=o(\log n)$; hence algorithms can afford to be exponential in the dimension. In the low-dimensional regime, efficient ANN algorithms are known for any metric space [16, 19, 29, 31]. In this paper, we focus on the high-dimensional regime, when $\omega(\log n) \leq$ $d \leq n^{o(1)}$, which is relevant for many applications.

The most studied metrics are the Hamming $\left(\ell_{1}\right)$ and the Euclidean $\left(\ell_{2}\right)$ distances. There are good reasons for this: $\ell_{1}$ and $\ell_{2}$ are very common in applications and admit very efficient algorithms based on hashing, in particular, Locality-Sensitive Hashing (LSH) [5, 26] and its data-dependent versions [7, 11]. Hashing-based algorithms for ANN over $\ell_{1} / \ell_{2}$ have now been the subject of a two-decade-long line of work, leading to a very good understanding of algorithms and their limitations. All such algorithms for $c$-approximate ANN obtain space $n^{1+\rho_{u}+o(1)}$ and query time $n^{\rho_{q}+o(1)}$ for some exponents $\rho_{u}$ and $\rho_{q}<1$ dependent on $c$; e.g., the most recent paper [9] gives tight time-space trade-offs for every approximation factor $c>1{ }^{1}$ We point the reader to [22] and [9], which summarize the state of affairs of the high-dimensional ANN over $\ell_{1} / \ell_{2}$. A practical perspective is presented in the surveys [40,41].

Beyond $\ell_{1}$ and $\ell_{2}$, the landscape of ANN is much more mysterious, despite having received significant attention. In 1998, [23] showed an efficient data structure for $\ell_{\infty}$ for approximation $c=$ $O(\log \log d)$. There are a few extensions of this result to other metrics, some of which proceed via embedding a metric into $\ell_{\infty}$ (see Section 1.3). However, we are still very far from having a general recipe for ANN data structures for general metrics with a non-trivial approximation; this is in stark contrast with the success of the lowdimensional regime. This state of affairs motivates the following broad question.

Problem 1. For a given approximation $c>1$, which metric spaces admit efficient ANN algorithms?

An algorithm for general metrics is highly desirable both in theory and in practice. From the theoretical perspective, we are interested in a common theory of ANN algorithms for a wide class of

\footnotetext{
${ }^{1}$ The exact dependence, for $\ell_{2}$, is that one can achieve any $\rho_{u}, \rho_{q} \geq 0$ satisfying $c^{2} \sqrt{\rho_{q}}+\left(c^{2}-1\right) \sqrt{\rho_{u}}=\sqrt{2 c^{2}-1}$.
} 
distances. Such a theory would yield data structures (or impossibility results) for a variety of important distance measures for which we still do not know efficient ANN algorithms (e.g., matrix norms, the Earth Mover's Distance (EMD), the edit distance, etc.). Perhaps even more tantalizing is understanding what exactly makes some distances harder than others, and how to quantify that hardness. From the practical perspective, it is also desirable to have a generic algorithm: one that either uses the underlying distance measure as a black box, or provides a "knob" to easily specialize to any desired distance measure. In practice, one must oftentimes tune the distance to the specifics of the application, and hence algorithms that allow such tuning without major re-implementations are preferred.

In this paper, we focus on the following important case of Problem 1.

Problem 2. Solve Problem 1 for high-dimensional normed spaces.

Norms are important for two reasons. First, most metric spaces arising in applications are actually norms (e.g., the Earth-Mover Distance [38]). Second, norms are geometrically nicer than general metrics, so there is hope for a coherent theory (e.g., for the problems of sketching and streaming norms, see the generic results of $[8,17])$. Using embeddings into $\ell_{2}[13,27]$, one can solve ANN for any norm with approximation $O(\sqrt{d / \varepsilon})$, space $n^{1+\varepsilon}$, and query time $n^{\varepsilon}$, where $0<\varepsilon<1 / 2$ is a constant; however, no better results are known in general.

\subsection{Our Main Result}

In this paper we nearly settle Problem 2 for symmetric norms, i.e., norms that are invariant under all permutations and changes of signs of the coordinates of a vector. We show the following general result:

THEOREM 1.1. For every $n \geq 1, d=n^{o(1)}$, and everyd-dimensional symmetric norm $\|\cdot\|$, there exists a data structure for ANN over $\|\cdot\|$ for $n$-point datasets with approximation $(\log \log n)^{O(1)}$, space $n^{1+o(1)}$, and query time $n^{o(1)}$.

We note that the techniques behind Theorem 1.1 cannot be extended to general norms; see details in Section 1.6.

\subsection{Why Symmetric Norms?}

The class of symmetric norms is, in some sense, a sweet spot. On the one hand, symmetric norms are mathematically nice and, as we show, allow for a clean characterization that leads to an efficient ANN data structure (see the proof overview from Section 1.4). On the other hand, symmetric norms vastly generalize $\ell_{p}$ distances and enable many new interesting examples, some of which arise in applications. We first consider the following two examples of symmetric norms, which are crucial for the subsequent discussion.

The first important example is the top- $k$ norm: the sum of $k$ largest absolute values of the coordinates of a vector; $k=1$ corresponds to $\ell_{\infty}$, while $k=d$ corresponds to $\ell_{1}$. Another rich set of examples is that of Orlicz norms: for any non-zero convex function $G: \mathbb{R}_{+} \rightarrow \mathbb{R}_{+}$such that $G(0)=0$, we define the unit ball of a norm $\|\cdot\|_{G}$ to be:

$$
\left\{x \in \mathbb{R}^{d} \mid \sum_{i=1}^{d} G\left(\left|x_{i}\right|\right) \leq 1\right\}
$$

Clearly, for $1 \leq p<\infty$ the $\ell_{p}$ norm is Orlicz via $G(t)=t^{p}$.

In statistics and machine learning, Orlicz norms are known as $M$-estimators (for the case of convex losses) [20]. A specific example is the Huber loss. Even though non-convex losses do not correspond to norms, our algorithm still can handle them (see Section 3).

Other examples of symmetric norms used in applications include:

- $k$-support norm [12] used for the sparse regression problem; its unit ball is the convex hull of $\left\{x \mid x\right.$ is $k$-sparse, $\|x\|_{2} \leq$ $1\}$,

- box- $\Theta$ norm [35] (again, used for sparse regression), defined for $0<a<b \leq c$ and $\Theta=\left\{\theta \in[a, b]^{d} \mid\|\theta\|_{1} \leq c\right\}$ as $\|x\|=\min _{\theta \in \Theta}\left(\sum_{i=1}^{d} \frac{x_{i}^{2}}{\theta_{i}}\right)^{1 / 2}$, and its dual;

- $K$-functional [21] used to show tight tail bounds, defined for $t>0$ as $\|x\|=\min \left\{\left\|x_{1}\right\|_{1}+t \cdot\left\|x_{2}\right\|_{2} \mid x_{1}+x_{2}=x\right\}$,

- $\|\cdot\|_{1,2, s}$ norms [30] used for dimension reduction, defined as $\|x\|=\left(\sum_{i}\left\|x_{S_{i}}\right\|_{1}^{2}\right)^{1 / 2}$, where $S_{1}$ is the set of $s$ largest absolute values of coordinates of $x, S_{2}$ is the set of next $s$ largest coordinates, etc.

Finally, we show two simple ways to construct many interesting examples of symmetric norms. Let $0=a_{0} \leq a_{1} \leq a_{2} \leq \ldots \leq a_{d}$ be a non-decreasing sub-additive ${ }^{2}$ sequence. We can define two norms associated with it [15]: a minimal norm of $x \in \mathbb{R}^{d}$ is defined as

$\max _{1 \leq k \leq d} a_{k} \cdot($ avg of largest $k$ absolute values of coordinates of $x$ ), and a maximal norm of $x \in \mathbb{R}^{d}$ is equal to

$\sum_{k=1}^{d}\left(a_{k}-a_{k-1}\right) \cdot(k$-th largest absolute value of a coordinate of $x)$.

The minimal norm is the smallest norm such that for every $k \in[d]$, one has:

$$
\|(\underbrace{1,1, \ldots, 1}_{k}, 0,0, \ldots, 0)\|=a_{k} .
$$

Similarly, the maximal norm is the largest such norm. Minimal norms will provide hard examples of symmetric norms that preclude some simple(r) approaches to ANN (see Section B.1). We also note that the dual (with respect to the standard dot product) of any symmetric norm is symmetric as well.

\subsection{Prior Work: ANN for Norms Beyond $\ell_{1}$ and $\ell_{2}$}

For norms beyond $\ell_{1}$ and $\ell_{2}$, the cornerstone result in ANN is a data structure for $\ell_{\infty}$ due to Indyk [23]. For every $\varepsilon>0$, the data structure achieves space $n^{1+\varepsilon}$, query time $n^{o(1)}$, and approximation $O_{\varepsilon}(\log \log d)$. This is a doubly-exponential improvement over embeddings of $\ell_{\infty}$ into $\ell_{1} / \ell_{2}$ which require distortion $\Omega(\sqrt{d})$.

It is well-known [42] that any $d$-dimensional normed space embeds into $\ell_{\infty}$ with distortion $(1+\varepsilon)$, which raises the question: can we combine this embedding with the result from [23] to solve ANN for any norm? It turns out that the answer is negative: accommodating a norm of interest may require embedding into a very

${ }^{2}$ For every $n, m$, one has $a_{n+m} \leq a_{n}+a_{m}$. 
high-dimensional $\ell_{\infty}$. In the worst case, we need $2^{O_{\varepsilon}(d)}$ dimensions, and this bound is known to be tight [13], even for spaces as simple as $\ell_{2}$. Even though this approach would give a non-trivial approximation of $O\left(\log \log 2^{O(d)}\right)=O(\log d)$, the resulting data structure has query time which is exponential in $d$; thus, this approach is interesting only for the low-dimensional regime $d=o(\log n)$.

The result of [23] has been extended as follows. In [2, 6, 24, 25] it was shown how to build data structures for ANN over arbitrary $\ell_{p}$-products of metrics given that there exists an ANN data structure for every factor. Recall that the $\ell_{p}$-product of metric spaces $M_{1}, M_{2}$, $\ldots, M_{k}$ is a metric space with the ground set $M_{1} \times M_{2} \times \ldots \times M_{k}$ and the following distance function:

$$
\begin{aligned}
& d\left(\left(x_{1}, x_{2}, \ldots, x_{k}\right),\left(y_{1}, y_{2}, \ldots, y_{k}\right)\right)= \\
& \left\|\left(d_{M_{1}}\left(x_{1}, y_{1}\right), d_{M_{2}}\left(x_{2}, y_{2}\right), \ldots, d_{M_{k}}\left(x_{k}, y_{k}\right)\right)\right\|_{p} .
\end{aligned}
$$

In a nutshell, if we can build efficient ANN data structures for every $M_{i}$ with approximation $c$, there exist an efficient data structure for ANN over the product space with approximation $O(c \cdot \log \log n)$. Note that the above also implies ANN for the standard $\ell_{p}$, though for this case a better approximation $O(\log \log d)$ is possible via randomized embeddings into $\ell_{\infty}$ [2].

For small values of $p$, one can also get $c=2^{O(p)}[14,37]$ using different techniques.

\subsection{Overview of the Proof of Theorem 1.1}

We prove Theorem 1.1 in three steps.

- First, we build a data structure for $d$-dimensional top- $k$ norms. We proceed by constructing a randomized embedding into $d$-dimensional $\ell_{\infty}$ with constant distortion, and then invoke the data structure for ANN over $\ell_{\infty}$ from [23].

Our embedding is a refinement of the technique of max$p$-stable distributions used in [2] to embed $\ell_{p}$ into $\ell_{\infty}$. Surprisingly, the technique turns out to be very general, and can handle top- $k$ norms as well an arbitrary Orlicz norm.

While this technique can handle even arbitrary symmetric norms (see Appendix B), there exist symmetric norms, for which this approach leads to merely a $\log ^{\Omega(1)} d$-approximation, which is exponentially worse than the bound we are aiming at (see Section B.1).

- To bypass the above limitation and obtain the desired $(\log \log n)^{O(1)}$-approximation, we show the following structural result: any $d$-dimensional symmetric norm allows a constant-distortion (deterministic) embedding into a lowdimensional iterated product of top- $k$ norms. More specifically, the host space $Y$ is an $\ell_{\infty}$-product of $d^{O(1)}$ copies of the $\ell_{1}$-product of $X_{1}, X_{2}, \ldots, X_{d}$, where $X_{k}$ is $\mathbb{R}^{d}$ equipped with the top- $k$ norm.

The dimension of $Y$ is $d^{O(1)}$ which is significantly better than the bound $2^{\Omega(d)}$ necessary to embed symmetric norms (even $\ell_{2}$ ) into $\ell_{\infty}$. It is exactly this improvement over the naïve approach that allows us to handle any dimension $d=n^{o(1)}$ as opposed to the trivial $o(\log n)$.

- Finally, we use known results [2, 24], which allow us to construct a data structure for ANN over a product space if we have ANN data structures for the individual factors.
Each such step incurs an additional $\log \log n$ factor in the resulting approximation. Since we have built a data structure for top- $k$ norms, and can embed a symmetric norm into an iterated product of top- $k$ norms, we are done!

Embeddings into iterated product spaces have been successfully used before for constructing data structures for ANN over Fréchet distance [24], edit distance [25], and Ulam distance [6]. Theorem 1.1 gives yet another confirmation of the power of the technique.

\subsection{Optimality of Theorem 1.1}

There remains one aspects of Theorem 1.1 that can potentially be improved: the approximation factor $(\log \log n)^{O(1)}$.

One of the bottlenecks for our algorithm is the ANN data structure for $\ell_{\infty}$ from [23], which gives $O(\log \log d)$ approximation. This bound is known to be tight $[4,28]$ for certain models of computation (in particular, for decision trees, which captures the result of [23]). Thus, going beyond approximation $\Omega(\log \log d)$ in Theorem 1.1 might be hard; however, it remains entirely possible to improve the approximation from $(\log \log n)^{O(1)}$ to $O(\log \log d)$, which we leave as an open question.

\subsection{Lower Bounds for General Norms}

The second step of the proof of Theorem 1.1 (see Section 1.4) shows how to embed any $d$-dimensional symmetric norm into a universal normed space of dimension $d^{O(1)}$ with a constant distortion. In contrast, we show that for general norms a similar universal construction is impossible. More formally, for a fixed $0<\varepsilon<1 / 3$, suppose $U$ is a normed space such that for every $d$-dimensional normed space $X$ there exists a randomized linear embedding of $X$ into $U$ with distortion $O\left(d^{1 / 2-\varepsilon}\right)$. Then, $U$ must have dimension at least $\exp \left(d^{\Omega_{\varepsilon}(1)}\right)$. By John's theorem [27], $d$-dimensional $\ell_{2}$ is a universal space for distortion $\sqrt{d}$, so our lower bound is tight up to sub-polynomial factors. See Section 6 for details.

To take this a step further, it would be highly desirable to prove stronger hardness results for ANN over general norms. One approach would be to show that such a norm $X$ has high robust expansion, which is a property used to deduce ANN lower bounds $[9,39]$. There exist metrics $M$ that have high robust expansion, such as the shortest path metric of a spectral expander (see Appendix C). To obtain a hard norm, it suffices to embed such an $N$-point metric $M$ into a $\log ^{O(1)} N$-dimensional norm with a constant distortion. The result of [34] shows that there exist $N$-point metrics $M$ which cannot be embedded into any norm of dimension $N^{o(1)}$. However, these metrics are not expanders, and for expanders such a dimension reduction procedure might be possible. Nonetheless, following the announcement of a draft of this paper, this question has been resolved negatively in the recent work of Naor [36]. In particular, Naor shows that embedding an $N$-point spectral expander with constant distortion into any normed space requires $N^{\Omega(1)}$ dimensions. 


\subsection{Other Related Work: Dealing with General Norms}

The recent result of [17] completely characterizes the streaming complexity of any symmetric norm. Even though many symmetric norms (including $\ell_{\infty}$ ) are hard in the streaming model, the state of affairs with ANN is arguably much nicer. In particular, our results imply that all symmetric norms have highly efficient ANN data structures. We also point out that streaming algorithms for the special case of Orlicz norms have been studied earlier [18].

Another related work is [8], which shows that for norms, the existence of good sketches is equivalent to uniform embeddability into $\ell_{2}$. Sketches are known to imply efficient ANN data structures, but since many symmetric norms do not embed into $\ell_{2}$ uniformly, we conclude that ANN is provably easier than sketching for a large class of norms.

Finally, we also mention the work of [1], who study ANN under the class of high-dimensional distances which are Bregman divergences. These results are somewhat disjoint since the Bregman divergences are not norms.

Acknowledgments. We thank Piotr Indyk for suggesting the proof of Theorem 6.1. We also thank Assaf Naor for discussions related to these research questions. Thanks to Clément Canonne for pointing us to relevant literature about symmetric norms.

This work is supported in part by grants from Simons Foundation (\#491119 to Alexandr Andoni), Google Faculty Research Award, and NSF (CCF-1617955), as well as by the NSF Graduate Research Fellowship (Grant No. DGE-16-44869).

\section{PRELIMINARIES}

\subsection{Norms and Products}

We denote non-negative real numbers by $\mathbb{R}_{+}$. For any subset $A \subseteq \mathbb{R}$, we let $\chi_{A}: \mathbb{R} \rightarrow\{0,1\}$ be the indicator function of $A$. Let $X$ be a normed space over $\mathbb{R}^{d}$. We denote $B_{X}$ the unit ball of $X$, and $\|\cdot\|_{X}$ the norm of $X$. We denote $X^{*}$ the dual norm of $X$ with respect to the standard dot product $\langle\cdot, \cdot\rangle$, i.e $\|x\|_{X^{*}}=\sup \left\{|\langle x, y\rangle|: y \in B_{X}\right\}$. For a vector $x \in \mathbb{R}^{d}$ we define $|x|=\left(\left|x_{1}\right|,\left|x_{2}\right|, \ldots,\left|x_{d}\right|\right)$ to be the vector of the absolute values of the coordinates of $x$. For a positive integer $d$ and $1 \leq p \leq \infty$, we denote $\ell_{p}^{d}$ the space $\mathbb{R}^{d}$ equipped with the standard $\ell_{p}$ norm, which we denote by $\|\cdot\|_{p}$.

Definition 2.1. For any vector $x \in \mathbb{R}^{d}$, we let $x^{*}=P|x|$ be the vector obtained by applying the permutation matrix $P$ to $|x|$ so coordinates of $x^{*}$ are sorted in non-increasing absolute value.

Definition 2.2 (Symmetric norm). A norm $\|\cdot\|_{X}: \mathbb{R}^{d} \rightarrow \mathbb{R}$ is symmetric if for every $x \in \mathbb{R}^{d},\|x\|_{X}=\||x|\|_{X}=\left\|x^{*}\right\|_{X}$.

See the introduction for examples of symmetric norms. We note once again that the dual norm of a symmetric norm is also symmetric.

A natural way to combine norms is via product spaces, which we will heavily exploit in this paper.

Definition 2.3 (Product space). Let $1 \leq p \leq \infty$. Let $\left(X_{1}, d_{X_{1}}\right)$, $\left(X_{2}, d_{X_{2}}\right), \ldots,\left(X_{k}, d_{X_{k}}\right)$ be metric spaces. We define the $\ell_{p}$-product space, denoted $\bigoplus_{\ell_{p}} X_{i}$, to be a metric space whose ground set is
$X_{1} \times X_{2} \times \ldots \times X_{k}$, and the distance function is defined as follows: the distance between $\left(x_{1}, x_{2}, \ldots, x_{k}\right)$ and $\left(x_{1}^{\prime}, x_{2}^{\prime}, \ldots, x_{k}^{\prime}\right)$ is defined as the $\ell_{p}$ norm of the vector $\left(d_{X_{1}}\left(x_{1}, x_{1}^{\prime}\right), d_{X_{2}}\left(x_{1}, x_{2}^{\prime}\right), \ldots, d_{X_{k}}\left(x_{k}, x_{k}^{\prime}\right)\right)$.

Next we define the top- $k$ norm:

Definition 2.4. For any $k \in[d]$, the top- $k$ norm, $\|\cdot\|_{T(k)}: \mathbb{R}^{d} \rightarrow \mathbb{R}$, is the sum of the absolute values of the top $k$ coordinates. In other words,

$$
\|x\|_{T(k)}=\sum_{i=1}^{k}\left|x_{i}^{*}\right|,
$$

where $x^{*}$ is the vector obtained in Definition 2.1.

\subsection{ANN for $\ell_{\infty}$ and $\ell_{\infty}$-products}

We will crucially use the following two powerful results of Indyk. The first result is for the standard $d$-dimensional $\ell_{\infty}$ space.

Theorem 2.5 ([23, Theorem 1]). For any $\varepsilon \in(0,1 / 2)$, there exists a data structure for ANN for $n$-points datasets in the $\ell_{\infty}^{d}$ space with approximation $O\left(\frac{\log \log d}{\varepsilon}\right)$, space $O\left(d \cdot n^{1+\varepsilon}\right)$, and query time $O(d$. $\log n)$.

The second is a generalization of the above theorem, which applies to an $\ell_{\infty}$-product of $k$ metrics $X_{1}, \ldots X_{k}$, and achieves approximation $O(\log \log n)$. It only needs black-box ANN schemes for each metric $X_{i}$.

Theorem 2.6 ([24, Theorem 1]). Let $X_{1}, X_{2}, \ldots, X_{k}$ be metric space, and let $c>1$ be a real number. Suppose that for every $1 \leq i \leq k$ and every $n$ there exists a data structure for ANN for $n$-point datasets from $X_{i}$ with approximation c, space $S(n) \geq n$, query time $Q(n)$, and probability of success 0.99 . Then, for every $\varepsilon>0$, there exists ANN under $\bigoplus_{\ell_{\infty}}^{k} \mathcal{M}$ with:

- $O\left(\varepsilon^{-1} \log \log n\right)$ approximation,

- $O(Q(n) \log n+d k \log n)$ query time, where d is the time to compute distances in each $X_{i}$, and

- $S(n) \cdot O\left(k n^{\varepsilon}\right)$ space/preprocessing.

Strictly speaking, we need to impose a technical condition on the ANN for each $X_{i}$ - that it reports the point with the smallest priority - which is satisfied in all our scenarios; see [24, Section 2] for details. Also, the original statement of [24] gave a somewhat worse space bound. The better space results simply from a better analysis of the algorithm, as was observed in [6]; we include a proof in Appendix A.

\section{ANN FOR ORLICZ AND TOP- $K$ NORMS}

Before showing a data structure for general symmetric norms, we give an algorithm for general Orlicz norms. We then show how to apply these ideas to top- $k$ norms. This restricted setting has a simple analysis and illustrates one of the main techniques used in the rest of the paper. A similar approach was used in prior work to construct randomized embeddings of $\ell_{p}$ norms into $\ell_{\infty}$, and solve the ANN search problem; here we show that these techniques are in fact applicable in much greater generality.

Lemma 3.1. Let $\|\cdot\|_{G}$ be an Orlicz norm. For every $D, \alpha>1$ and every $\mu \in(0,1 / 2)$ there exists a randomized linear map $f: \mathbb{R}^{d} \rightarrow \mathbb{R}^{d}$ such that for every $x \in \mathbb{R}^{d}$ : 
- if $\|x\|_{G} \leq 1$, then $\operatorname{Pr}_{f}\left[\|f(x)\|_{\infty} \leq 1\right] \geq \mu$;

- if $\|x\|_{G}>\alpha D$, then $\operatorname{Pr}_{f}\left[\|f(x)\|_{\infty}>D\right] \geq 1-\mu^{\alpha}$.

Proof. Let the distribution $\mathcal{D}$ over $\mathbb{R}_{+}$have the following CDF $F: \mathbb{R}_{+} \rightarrow[0,1]:$

$$
F(t)=\operatorname{Pr}_{u \sim \mathcal{D}}[u \leq t]=1-\mu^{G(t)} .
$$

Consider the following randomized linear map $f: \mathbb{R}^{d} \rightarrow \mathbb{R}^{d}$ :

$$
\left(x_{1}, x_{2}, \ldots, x_{d}\right) \stackrel{f}{\mapsto}\left(\frac{x_{1}}{u_{1}}, \frac{x_{2}}{u_{2}}, \ldots, \frac{x_{d}}{u_{d}}\right)
$$

where $u_{1}, \ldots, u_{d} \sim \mathcal{D}$ are i.i.d. samples from $\mathcal{D}$. Suppose that $\|x\|_{G} \leq 1$. Then, $\sum_{i=1}^{d} G\left(\left|x_{i}\right|\right) \leq 1$. This, in turn, implies:

$$
\begin{aligned}
\operatorname{Pr}_{f}\left[\|f(x)\|_{\infty} \leq 1\right] & =\prod_{i=1}^{d} \underset{u_{i} \sim \mathcal{D}}{\operatorname{Pr}}\left[\left|\frac{x_{i}}{u_{i}}\right| \leq 1\right]=\prod_{i=1}^{d} \mu^{G\left(\left|x_{i}\right|\right)} \\
& =\mu^{\sum_{i=1}^{d} G\left(\left|x_{i}\right|\right)} \geq \mu .
\end{aligned}
$$

Now suppose that $\|x\|_{G}>\alpha D$. This, together with the convexity of $G(\cdot)$, implies:

$$
\sum_{i=1}^{d} G\left(\frac{\left|x_{i}\right|}{D}\right) \geq(1-\alpha) G(0)+\alpha \cdot \sum_{i=1}^{d} G\left(\frac{\left|x_{i}\right|}{\alpha D}\right) \geq \alpha .
$$

Thus, we have:

$$
\begin{aligned}
\operatorname{Pr}_{f}\left[\|f(x)\|_{\infty} \leq D\right] & =\prod_{i=1}^{d} \operatorname{Pr}_{u_{i} \sim \mathcal{D}}\left[\left|\frac{x_{i}}{u_{i}}\right| \leq D\right]=\prod_{i=1}^{d} \mu^{G\left(\left|x_{i}\right| / D\right)} \\
& =\mu^{\sum_{i=1}^{d} G\left(\left|x_{i}\right| / D\right)} \leq \mu^{\alpha}
\end{aligned}
$$

Theorem 3.2. For every d-dimensional Orlicz norm $\|\cdot\|_{G}$ and every $\varepsilon \in(0,1 / 2)$, there exists a data structure for ANN over $\|\cdot\|_{G}$, which achieves approximation $O\left(\frac{\log \log d}{\varepsilon^{2}}\right)$ using space $O\left(d n^{1+\varepsilon}\right)$ and query time $O\left(d n^{\varepsilon}\right)$.

Proof. Let $P \subset \mathbb{R}^{d}$ be a dataset of $n$ points. Consider the data structure which does the following:

(1) For all $1 \leq i \leq n^{\varepsilon}$, we independently apply the randomized linear map $f$ from Lemma 3.1 with parameters $\mu=n^{-\varepsilon}$, $D=O\left(\frac{\log \log d}{\varepsilon}\right)$, and $\alpha=\frac{2}{\varepsilon}$. We define

$$
P_{i}=\left\{f_{i}(x) \mid x \in P\right\}
$$

to be the image of the dataset under $f_{i}$, where $f_{i}$ is the $i$-th independent copy of $f$.

(2) For each $1 \leq i \leq n^{\varepsilon}$, we use Theorem 2.5 to build a data structure for ANN over $\ell_{\infty}$ with approximation $D$ for dataset $P_{i}$. We refer to the $i$-th data structure as $T_{i}$.

Each $T_{i}$ occupies space $O\left(d n^{1+\varepsilon}\right)$ and achieves approximation $D$ with query time $O(d \log n)$. To answer a query $q \in \mathbb{R}^{d}$, we query $T_{i}$ with $f_{i}(q)$ for each $i \in\left[n^{\varepsilon}\right]$. Let $x_{i}$ be the point returned by $T_{i}$, and let $p_{i} \in P$ be the pre-image of $x_{i}$ under $f_{i}$, so that $f_{i}\left(p_{i}\right)=x_{i}$. If for some $T_{i}$, the point returned satisfies $\left\|p_{i}-q\right\|_{G} \leq \alpha D$, then we return $p_{i}$.
- If there exists some $p \in P$ with $\|p-q\|_{G} \leq 1$, then by Lemma 3.1, with probability $1-\left(1-n^{-\varepsilon}\right)^{n^{\varepsilon}} \geq \frac{3}{5}$, some $f_{i}$ has $\left\|f_{i}(p-q)\right\|_{\infty} \leq 1$. Since $f_{i}$ is linear, $\left\|f_{i}(p)-f_{i}(q)\right\|_{\infty} \leq 1$ as well.

- Let $i \in\left[n^{\varepsilon}\right]$ be an index where some $p \in P$ with $\| p-$ $q \|_{G} \leq 1$ has $\left\|f_{i}(p)-f_{i}(q)\right\|_{\infty} \leq 1$. Every other $p^{\prime} \in P$ with $\left\|p^{\prime}-q\right\|_{G} \geq \alpha D$ satisfies

$$
\operatorname{Pr}\left[\left\|f_{i}\left(p^{\prime}\right)-f_{i}(q)\right\|_{\infty} \leq D\right] \leq \frac{1}{n^{2}} .
$$

A union bound over at most $n$ points with distance greater than $\alpha D$ to $q$ shows that except with probability at most $\frac{1}{n}, T_{i}$ returns some $p_{i} \in P$ with $\left\|p_{i}-q\right\|_{G} \leq \alpha D$. Thus, the total probability of success of the data structure is at least $\frac{3}{5}-\frac{1}{n}$.

The total query time is $O\left(d n^{\varepsilon} \cdot \log n\right)$ and the total space used is $O\left(d n^{1+2 \varepsilon}\right)$. This data structure achieves approximation $\alpha D=$ $O\left(\frac{\log \log d}{\varepsilon^{2}}\right)$. Decreasing $\varepsilon$ by a constant factor, we get the desired guarantees.

Remark. The construction of the randomized embedding in Lemma 3.1 and the data structure from Theorem 3.2 work in a somewhat more general setting, rather than just for Orlicz norms. For a fixed norm $\|\cdot\|$, we can build a randomized map $f: \mathbb{R}^{d} \rightarrow \mathbb{R}^{d}$ with the guarantees of Lemma 3.1 if there exists a non-decreasing $G: \mathbb{R}_{+} \rightarrow \mathbb{R}_{+}$where $G(0)=0, G(t) \rightarrow \infty$ as $t \rightarrow \infty$, and for every $x \in \mathbb{R}^{d}$ :

- if $\|x\| \leq 1$, then $\sum_{i=1}^{d} G\left(\left|x_{i}\right|\right) \leq 1$, and

- if $\|x\| \geq \alpha D$, then $\sum_{i=1}^{d} G\left(\frac{\left|x_{i}\right|}{D}\right) \geq \alpha$.

The data structure itself just requires the existence of a randomized linear map satisfying the conditions of Lemma 3.1.

We now describe how to obtain a data structure for ANN for any top- $k$ norm.

Lemma 3.3. Fix any $k \in[d]$. For every $D, \alpha>1$ and every $\mu \in$ $(0,1 / 2)$, there exists a randomized linear map $f: \mathbb{R}^{d} \rightarrow \mathbb{R}^{d}$ such that for every $x \in \mathbb{R}^{d}$ :

- if $\|x\|_{T(k)} \leq 1$, then $\operatorname{Pr}_{f}\left[\|f(x)\|_{\infty} \leq 1\right] \geq \mu$;

- if $\|x\|_{T(k)}>\alpha D$, then $\operatorname{Pr}_{f}\left[\|f(x)\|_{\infty}>D\right] \geq 1-\mu^{\alpha-1}$.

Proof. We define $G: \mathbb{R}_{+} \rightarrow \mathbb{R}_{+}$where for every $x \in \mathbb{R}^{d}$,

$$
G(t)=t \cdot \chi_{\left[\frac{1}{k}, \infty\right)}(t)
$$

If $\|x\|_{T(k)} \leq 1$, there are at most $k$ coordinates where $\left|x_{i}\right| \geq \frac{1}{k}$. Therefore, $\sum_{i=1}^{d} G\left(\left|x_{i}\right|\right) \leq\|x\|_{T(k)} \leq 1$. If $\|x\|_{T(k)} \geq \alpha D$, then $\sum_{i=1}^{k}\left|x_{i}^{*}\right| \geq \alpha D$. Therefore, $\sum_{i=1}^{d} G\left(\frac{\left|x_{i}^{*}\right|}{D}\right) \geq \sum_{i=1}^{k} G\left(\frac{\left|x_{i}^{*}\right|}{D}\right) \geq \alpha-1$. The proof now follows in the same way as Lemma 3.1.

Lemma 3.3 gives us a data structure for any top- $k$ norm with approximation $O(\log \log d)$ applying Theorem 3.2.

One could imagine using a similar argument to design an algorithm for general symmetric norms. This idea indeed works and yields an algorithm with approximation $\widetilde{O}(\log d)$ for a general symmetric norm (see Appendix B for a detailed analysis of this approach). However, we show this strategy cannot achieve 
an approximation better than $\Omega(\sqrt{\log d}$ ) (see the end of the same Appendix B).

\section{EMBEDDING SYMMETRIC NORMS INTO PRODUCT SPACES}

In this section, we construct an embedding of general symmetric norms into product spaces of top- $k$ norms. To state the main result of this section, we need the following definition.

Definition 4.1. For any $c_{1}, \ldots, c_{d} \geq 0$, let $\bigoplus_{\ell_{1}}^{d} T^{(c)} \subset \mathbb{R}^{d^{2}}$ be the space given by the seminorm $\|\cdot\|_{T, 1}^{(c)} \cdot \mathbb{R}^{d^{2}} \rightarrow \mathbb{R}$ where for $x=\left(x_{1}, \ldots, x_{d}\right) \in \mathbb{R}^{d^{2}}$ and $x_{1}, \ldots, x_{d} \in \mathbb{R}^{d}:$

$$
\|x\|_{T, 1}^{(c)}=\sum_{k=1}^{d} c_{k}\left\|x_{k}\right\|_{T(k)} .
$$

We prove the following theorem.

Theorem 4.2 (EMBEDding into A PRoduct SPACE). For any constant $\gamma \in(0,1 / 2)$, any symmetric norm $\|\cdot\|_{X}: \mathbb{R}^{d} \rightarrow \mathbb{R}$ can be embedded with distortion $1+\gamma$ into $\bigoplus_{\ell_{\infty}}^{t} \bigoplus_{\ell_{1}}^{d} T^{(c)}$ where $t=\left(\frac{d}{\gamma}\right)^{O\left(\gamma^{-1}\right)}$. In particular, there exists $c \in \mathbb{R}_{+}^{t \times d}$ such that for every $x \in \mathbb{R}^{d}$,

$$
(1-\gamma)\|x\|_{X} \leq \max _{i \in[t]}\left(\sum_{k=1}^{d} c_{i, k}\|x\|_{T(k)}\right) \leq(1+\gamma)\|x\|_{X}
$$

The vectors in $\bigoplus_{\ell_{\infty}}^{t} \bigoplus_{\ell_{1}}^{d} T^{(c)} \subset \mathbb{R}^{t d^{2}}$ can be broken up into $t d$ blocks of $d$ coordinates each. The embedding referenced above will simply map $x \in \mathbb{R}^{d}$ into $\mathbb{R}^{t d^{2}}$ by making each of the $t d$ many blocks equal to a copy of $x$. The non-trivial part of the above theorem is setting the constants $c_{i, k}$ for $i \in[t]$ and $k \in[d]$ so (1) holds. Theorem 4.2 can be stated more generally in terms of a function of the size of a certain $\gamma$-net. We need the following definitions.

Definition 4.3. Let the set $\mathcal{L} \subset \mathbb{R}_{+}^{d}$ be given by

$$
\mathcal{L}=\left\{y \in \mathbb{R}_{+}^{d} \mid y_{1} \geq \ldots y_{d} \geq 0\right\}
$$

Definition 4.4. For any fixed symmetric norm $\|\cdot\|_{X}: \mathbb{R}^{d} \rightarrow \mathbb{R}$ and $\gamma \in(0,1 / 2)$, we denote $\mathcal{E}(\gamma, X)$ as the size of the smallest $\gamma$-net of $B_{X^{*}} \cap \mathcal{L}$, where distances are measure with respect to the dual norm $X^{*}$

Given these definitions, we note that, in Theorem 4.2, it is enough to set $t$ to be $\mathcal{E}(\gamma, X)$. The proof of Theorem 4.2 follows from the following three lemmas.

Definition 4.5. Fix a vector $y \in \mathcal{L} \backslash\{0\}$ ( $y$ has non-negative, nonincreasing coordinates). Let the maximal norm with respect to $y$, $\|\cdot\|_{y}: \mathbb{R}^{d} \rightarrow \mathbb{R}$ be the norm where for every $x \in \mathbb{R}^{d}$,

$$
\|x\|_{y}=\left\langle\left|x^{*}\right|, y\right\rangle
$$

Lemma 4.6. For every vector $y \in \mathcal{L} \backslash\{0\}$, there exists $c_{1}, \ldots, c_{d} \geq 0$ where for all $x \in \mathbb{R}^{d}$,

$$
\|x\|_{y}=\|x\|_{T, 1}^{(c)}
$$

Proof. For $k \in[d]$, we let $c_{k}=y_{k}-y_{k+1}$, where $y_{d+1}=0$.

$$
\begin{aligned}
\left\langle\left|x^{*}\right|, y\right\rangle & =\sum_{i=1}^{d}\left|x_{i}^{*}\right| y_{i}=\sum_{i=1}^{d}\left|x_{i}^{*}\right|\left(\sum_{k=i}^{d} c_{k}\right) \\
& =\sum_{k=1}^{d} c_{k}\left(\sum_{i=1}^{k}\left|x_{i}^{*}\right|\right)=\sum_{k=1}^{d} c_{k}\|x\|_{T(k)}
\end{aligned}
$$

LEMMA 4.7. Fix an $\gamma \in(0,1 / 2)$. Let $\|\cdot\|_{X}$ be an arbitrary symmetric norm and $\|\cdot\|_{X^{*}}$ be its dual norm. If $N$ is an $\gamma$-net of $\mathcal{L} \cap B_{X^{*}}$, then

$$
(1-\gamma)\|x\|_{X} \leq \max _{y \in N}\|x\|_{y} \leq(1+\gamma)\|x\|_{X}
$$

Proof. Consider any fixed $x \in \mathbb{R}^{d}$. Then without loss of generality we may assume all coordinates of $x$ are non-negative and in non-increasing order. Then, because $X^{*}$ is symmetric,

$$
\|x\|_{X}=\max _{v \in B_{X^{*}}}\langle x, v\rangle=\max _{v \in B_{X^{*}} \cap \mathcal{L}}\langle x, v\rangle \leq \max _{y \in N}\langle x, y\rangle+\gamma\langle x, z\rangle,
$$

where $z \in B_{X^{*}}$. The second term above is bounded by $\|x\|_{X}$, so we have proved the first inequality. Likewise,

$$
\|x\|_{X} \geq \max _{y \in N}\langle x, y\rangle-\gamma\|x\|_{X}
$$

Finally, the last lemma establishes an upper bound on $\mathcal{E}(\gamma, X)$ (see Definition 4.4).

Lemma 4.8. For any $\gamma>0$, and any symmetric space $X$,

$$
\mathcal{E}(\gamma, X) \leq d^{O\left(\gamma^{-1} \log (1 / \gamma)\right)} .
$$

The proof of Theorem 4.2 is simply given by combining Lemma 4.6, Lemma 4.7, and Lemma 4.8 .

\subsection{Proof of Lemma 4.8: Bounding the $\gamma$-net Size}

We now give an upper bound on the size of the $\gamma$-net of $B_{X^{*}} \cap \mathcal{L}$. We let $\beta>1$ be a constant which we specify later ${ }^{3}$. Without loss of generality, we rescale the norm so that $\left\|e_{1}\right\|_{X^{*}}=1$, where $e_{1}$ is the first standard basis vector.

Definition 4.9. Given vectors $x, y \in \mathbb{R}^{d}$, we say $x$ weakly majorizes $y$ if for all $k \in[d]$,

$$
\sum_{i=1}^{k}\left|x_{i}^{*}\right| \geq \sum_{i=1}^{k}\left|y_{i}^{*}\right|
$$

Lemma 4.10 (Theorem B.2 In [33]). If $x, y \in \mathbb{R}^{d}$ where $x$ majorizes $y$, then for any symmetric norm $\|\cdot\|_{X}$,

$$
\|x\|_{X} \geq\|y\|_{X} .
$$

Definition 4.11. For $i \in[d]$, let $\xi^{(i)} \in \mathbb{R}^{d}$ be the vector

$$
\xi^{(i)}=(\underbrace{1, \ldots, 1}_{i}, \underbrace{0, \ldots, 0}_{d-i})
$$

consisting of exactly $i 1$ 's, and $d-i 0$ 's.

${ }^{3}$ We will eventually set $\beta$ to $1+\frac{1}{100}$ in order to get an embedding with $\gamma=\frac{1}{10}$ in the algorithm. 
Definition 4.12 (Levels and Level Vectors). For any fixed vector $x \in \mathbb{R}^{d}$, we define level $k$ with respect to $x$ as $B_{k}=\{i \in[d] \mid$ $\left.\beta^{-k-1} \leq\left|x_{i}\right| \leq \beta^{-k}\right\}$. Additionally, we let $b_{k}=\left|B_{k}\right|$ be the size of level $k$ with respect to $x$. The level vector of $x, V(x) \in \mathbb{R}^{d}$ is given by

$$
V(x)=(\underbrace{\beta^{0}, \ldots, \beta^{0}}_{b_{0} \text { times }}, \underbrace{\beta^{-1}, \ldots, \beta^{-1}}_{b_{1} \text { times }}, \ldots, \underbrace{\beta^{-t}, \ldots, \beta^{-t}}_{b_{t} \text { times }}, 0, \ldots 0)
$$

and the $i$-th level vector $V_{i}(x) \in \mathbb{R}^{d}$ is given by

$$
V_{i}(x)=(\underbrace{\beta^{-i}, \ldots, \beta^{-i}}_{b_{i} \text { times }}, 0, \ldots, 0) .
$$

The notation used for level vectors appears in [17]; however, we refer to level $k$ as the coordinates of $x$ lying in $\left(\beta^{-k-1}, \beta^{-k}\right.$; whereas [17] refers to level $k$ as the coordinates of $x$ lying in $\left[\beta^{k-1}, \beta^{k}\right)$.

Definition 4.13. Fix some $\tau>0$. For any vector $x \in \mathbb{R}^{d}$, let $C(x) \in \mathbb{R}^{d}$ be the vector where each $i \in[d]$,

$$
C(x)_{i}=\left\{\begin{array}{cc}
x_{i} & \left|x_{i}\right| \geq \tau \\
0 & \left|x_{i}\right|<\tau
\end{array} .\right.
$$

Proposition 4.14 (Proposition 3.4 In [17]). Let $\|\cdot\|_{X}$ be any symmetric norm and $x \in \mathbb{R}^{d}$ be any vector. Then

$$
\frac{1}{\beta}\|V(x)\|_{X} \leq\|x\|_{X} \leq\|V(x)\|_{X}
$$

Proposition 4.15. Let $\|\cdot\|_{X}$ be any symmetric norm. For any vector $x \in \mathbb{R}^{d}$,

$$
\|x\|_{X}-\tau d \leq\|C(x)\|_{X} \leq\|x\|_{X}
$$

Proof. Note that $x$ weakly majorizes $C(x)$, so $\|C(x)\|_{X} \leq\|x\|_{X}$ For the other direction, let $v=x-C(x)$. Then $\tau d \xi^{(1)}$ weakly majorizes $v$. Therefore,

$$
\|x\|_{X} \leq\|C(x)\|_{X}+\|v\|_{X} \leq\|C(x)\|_{X}+\tau d
$$

Intuitively, the above two propositions say that up to multiplicative loss $\beta$ and additive loss $\tau d$ in the norm of the vector, we may assume that all coordinates are exactly $\beta^{j}$ for $j \leq \log _{\beta}\left(\frac{1}{\tau}\right)$. Thus, if $x \in \mathbb{R}^{d}$, then

$$
\|x\|_{X}-\tau d \leq\|V(C(x))\|_{X} \leq \beta\|x\|_{X}
$$

If additionally, $\tau=\frac{\beta}{d^{2}}$, and $\|x\|_{X} \geq \frac{2}{d}$,

$$
\|x\|_{X} \leq\|2 V(C(x))\|_{X} \leq 2 \beta\|x\|_{X}
$$

and there are at most $2 \log _{\beta} d$ non-empty levels in $V(C(x))$. In order to build the $\gamma$-net, we define a more refined representation of vectors.

Definition 4.16 (Rounded counts vector). Fix any level vector $x \in$ $\mathbb{R}^{d}$ and let $b_{k}$ be the number of coordinates of $x$ in level $k$. The rounded counts vector of $x, R(x) \in \mathbb{R}^{d}$ is given by $y$ where the $y \in \mathbb{R}^{d}$ is constructed using the following procedure:

(1) Iterate over $k=-\infty, \ldots, 2 \log _{\beta} d-1$, maintaining a vector $y \in \mathbb{R}^{d}$, where initially $y=0$. Let $c$ be the number of coordinates which are 0 in $y$, so initially, $c=d$.
(2) If $b_{k} \neq 0$, let $j \in \mathbb{Z}_{+}$be the integer where $\beta^{j-1}<b_{k} \leq \beta^{j}$. If $c \geq\left\lfloor\beta^{j}\right\rfloor$, then replace $\left\lfloor\beta^{j}\right\rfloor$ zero-coordinates in $y$ with $\beta^{-k}$.

(3) Repeat the above procedure, increasing $k$, until $c=0$ or $k=2 \log _{\beta} d-1$.

Intuitively, $R(x)$ represents the level vector of $x$ where we ignore coordinates smaller than $\frac{\beta}{d^{2}}$, and additionally, we round the counts of coordinates to powers of $\beta$.

Next we construct a further simplification of $R(x)$. Intuitively we show that one can ignore the higher levels if there are fewer coordinates in the higher levels than some lower level.

Lemma 4.17. Let $\|\cdot\|_{X}$ be a symmetric norm. Consider nonnegative vectors $x, y \in \mathbb{R}_{+}^{d}$ and two indices $u, v \in[d]$ such that $x$ and $y$ agree on all coordinates except for $u$ and $v\left(y_{k}=x_{k} \forall k \neq u, v\right)$ and $y_{u}=x_{u}+x_{v}, y_{v}=0$. Then $\|y\|_{X} \geq\|x\|_{X}$.

Proof. Assume that $\|x\|_{X}=\langle x, z\rangle$ where $z$ is a nonnegative vector in $B_{X^{*}}$. Consider vector $z^{\prime}$ such that $z_{k}^{\prime}=z_{k} \forall k \neq u, v$ and $z_{u}^{\prime}=\max \left(z_{u}, z_{v}\right), z_{v}^{\prime}=\min \left(z_{u}, z_{v}\right)$. Because $X^{*}$ is symmetric and $z^{\prime}$ is just a permutation of $z$, we have $z^{\prime} \in B_{X^{*}}$. Also notice that $z_{u}^{\prime} y_{u}+z_{v}^{\prime} y_{v}=\max \left(z_{u}, z_{v}\right)\left(x_{u}+x_{v}\right) \geq z_{u} x_{u}+z_{v} x_{v}$. Therefore, $\|y\|_{X} \geq\left\langle y, z^{\prime}\right\rangle \geq\langle x, z\rangle$.

Definition 4.18. Consider a vector $x \in \mathbb{R}^{d}$. We define the simplified rounded vector $S(x)$ as the vector $z$ of the following procedure.

1: Initialize $z=R(V(x))$

2: for $i=0,1, \ldots, 2 \log _{\beta}(d)-1$ do

3: $\quad$ if $b_{i}(z) \leq(\beta-1)^{2} \max _{j<i+\log _{\beta}(\beta-1)} b_{j}(z)$ then

4: $\quad$ Set all coordinates of $z$ of value $\beta^{-i}$ to 0 i.e. set $b_{i}(z)=0$. end if

6: end for

7: Sort the coordinates of $z$ in non-increasing order.

Next we show that the simplified rounded vector is close to the rounded counts vector.

Lemma 4.19. Let $\|\cdot\|_{X}$ be a symmetric norm. Consider a rounded counts vector $x \in \mathbb{R}_{+}^{d}$. We have $\|S(x)-x\|_{X} \leq(\beta-1)\left(\beta^{2}+1\right)\|x\|_{X}$.

Proof. The number of coordinates of $x$ in level $i$ that are not equal to the same coordinates in $z$ is at most the number of coordinates zeroed out in all levels $j \leq i$ (causing the shifting of coordinates). Thus, we can charge the non-zeros in $x-z$ to the zeroed out coordinates so that each zeroed out coordinate in level $j$ accounts for at most one difference involving a coordinate of $x$ in each level $i \geq j$. For each $j$, let $s_{j}<j+\log _{\beta}(\beta-1)$ be the index such that $(\beta-1)^{2} b_{s_{j}}(z) \geq b_{j}(z)$. We deliver all charges to zeroing out level $j$ to level $s_{j}$. All charges to the same level $k$ are from levels at least $k-\log _{\beta}(\beta-1)$ and they sum up to at most

$$
\begin{aligned}
\sum_{j \geq k-\log _{\beta}(\beta-1)}(\beta-1)^{2} b_{k}(z) \sum_{i \geq j} \beta^{-i} \\
\leq \sum_{j \geq k-\log _{\beta}(\beta-1)}(\beta-1)^{2} b_{k}(z) \frac{\beta^{1-j}}{\beta-1} \\
\leq(\beta-1) b_{k}(z) \beta^{2-k}
\end{aligned}
$$


Thus we can divide the charges to the same level $k$ into $b_{k}(z)$ coordinates as equally as possible and each coordinate accounts for at most $(\beta-1) \beta^{2-k}+(\beta-1) \beta^{-k}$ (the average charge plus the maximum charge). By Lemma 4.17, aggregating these charges create a vector $y$ with $\|y\|_{X} \geq\|x-z\|_{X}$. However, $y$ is also majorized by $(\beta-1)\left(\beta^{2}+1\right) x$ so $\|x-z\|_{X} \leq(\beta-1)\left(\beta^{2}+1\right)\|x\|_{X}$.

Proof of Lemma 4.8. We prove the theorem by giving a $\gamma$-net of $\mathcal{L} \cap B_{X^{*}}$. Notice that each coordinate of any vector in $B_{X^{*}}$ is at most 1 in absolute value, since $x^{*}$ weakly majorizes $x_{1}^{*} e_{1}$, and we assumed that $\left\|e_{1}\right\|_{X^{*}}=1$. Let $\beta=1+\frac{\gamma}{10}$, and consider the set

$$
N=\left\{S(x) \mid x \in \mathcal{L} \cap B_{X^{*}}\right\}
$$

First we bound from above the number of net points. Let $z=S(x)$ and let $t_{i}=\frac{b_{i}(z)}{\max _{j<i+\log _{\beta}(\beta-1)} b_{j}(z)}$. Let $i^{*}$ be the smallest $i$ with nonzero $b_{i}(z)$. For all $i>i^{*}-\log _{\beta}(\beta-1)$, we either have $t_{i}(z)=0$ or $t_{i}(z) \geq(\beta-1)^{2}$. Because $z$ has $d$ coordinates, we also have $\prod_{i=i^{*}-\log _{\beta}(\beta-1)}^{2 \log _{\beta} d-1} \max \left(1, t_{i}\right) \leq d^{-\log _{\beta}(\beta-1)}$.

To encode $z$, we just need to encode the values $b_{i}(z)$ as follows. Use $O\left(\log d \log _{\beta}(1 /(\beta-1))\right)$ bits to encode $i^{*}$ and $b_{i^{*}+j}(z)$ for $j=$ $0,1, \ldots,-\log _{\beta}(\beta-1)$. For each subsequent $i$ up to $2 \log _{\beta} d-1$, we can use 1 bit to encode whether $b_{i}(z)=0$ and if $b_{i}(z)>0$, additional $O\left(\log \log _{\beta} \frac{t_{i}}{(\beta-1)^{2}}\right)$ bits to encode $t_{i}$ using a prefix-free code (because $t_{i}$ is a power of $\beta$ that is at least $\left.(\beta-1)^{2}\right)$. Thus, the number of bits of the encoding is at most

$$
\begin{aligned}
& O\left(\log d \log _{\beta}(1 /(\beta-1))+\sum_{i>i^{*}-\log _{\beta}(\beta-1), t_{i}>0} \log \log _{\beta} \frac{t_{i}}{(\beta-1)^{2}}\right) \\
& =O\left(\left(\log _{\beta} d\right) \log \log _{\beta} \frac{1}{(\beta-1)^{2}}+\sum_{i>i^{*}-\log _{\beta}(\beta-1)} \log \max \left(1, t_{i}\right)\right) \\
& =O\left(\left(\log _{\beta} d\right) \log \log _{\beta} \frac{1}{(\beta-1)^{2}}\right)
\end{aligned}
$$

In other words, the size of the net is $d^{O(\log (1 / \gamma) / \gamma)}$.

For every $x \in \mathcal{L}, R(x)$ can have at most $\beta b_{k}$ coordinates at $\beta^{-k}$ in $R(x)$; whereas $x$ has $b_{k}$ coordinates at least $\beta^{-k-1}$. Thus, $R(x)-x$ has at most $(\beta-1) b_{k}$ coordinates at $\beta^{-k}$, and at most $b_{k}$ coordinates at most $\beta^{-k-1}(\beta-1)$. Therefore, $2(\beta-1) \beta x+\frac{\beta \xi^{(1)}}{d}$ weakly majorizes $R(x)-x$, and therefore, $\|R(x)-x\|_{X^{*}} \leq\|2(\beta-1) \beta x\|_{X^{*}}+\frac{\beta}{d} \leq$ $2(\beta-1) \beta+\frac{\beta}{d} \leq 5(\beta-1)$, for $1+\frac{1}{d-1}<\beta<2$.

Finally, by lemma $4.19,\|R(x)-S(x)\|_{X^{*}} \leq(\beta-1)\left(\beta^{2}+1\right)\|R(x)\|_{X^{*}} \leq$ $(\beta-1)\left(\beta^{2}+1\right) \beta^{2} \leq \gamma / 2$ for $\beta \leq 1.3$.

\section{PROOF OF THE MAIN THEOREM: ANN FOR SYMMETRIC NORMS}

We now prove our main result, Theorem 1.1. The algorithm here achieves approximation

$$
O\left(\frac{\log ^{2} \log n \cdot \log \log d}{\varepsilon^{5}}\right) .
$$

We proceed by giving an algorithm for $\bigoplus_{\ell_{\infty}}^{t} \bigoplus_{\ell_{1}}^{d} T^{(c)}$ using Theorem 2.5, Theorem 5.1.2 from [2], and Theorem 2.6.
Lemma 5.1. Fix some $c_{1}, \ldots, c_{d} \geq 0$. Let $\bigoplus_{\ell_{\infty}} T^{(c)}$ be the space with $\|\cdot\|_{T, \infty}^{(c)}: \mathbb{R}^{d^{2}} \rightarrow \mathbb{R}$ seminorm where for every $x=\left(x_{1}, \ldots, x_{d}\right) \in$ $\mathbb{R}^{d^{2}}$

$$
\|x\|_{T, \infty}^{(c)}=\max _{k \in[d]} c_{k}\left\|x_{k}\right\|_{T(k)} .
$$

For every $\varepsilon \in(0,1 / 2)$, there exists a data structure for ANN over $\|\cdot\|_{T, \infty}^{(c)}$ which achieves approximation $O\left(\frac{\log \log n \cdot \log \log d}{\varepsilon^{3}}\right)$ using space $O\left(d^{2} \cdot n^{1+\varepsilon}\right)$ and query time $O\left(d^{2} \cdot n^{\varepsilon}\right)$.

Proof. Given the randomized embedding from Lemma 3.3, we can build a data structure for $c_{k}\|\cdot\|_{T(k)}$ achieving approximation $O\left(\frac{\log \log d}{\varepsilon^{2}}\right)$ using space $O\left(d^{2} n^{1+\varepsilon / 2}\right)$ and query time $O\left(d^{2} n^{\varepsilon / 2}\right)$. This data structure works in the same way as in the proof of Theorem 3.2. We handle the constant $c_{k}$ by rescaling the norm, and since the embeddings are linear, it does not affect the correctness of the data structure. Then we apply Theorem 2.5.

Lemma 5.2. Fix some $c_{1}, \ldots, c_{d} \geq 0$. Let $\bigoplus_{\ell_{1}} T^{(c)}$ be the space with $\|\cdot\|_{T, 1}^{(c)}: \mathbb{R}^{d^{2}} \rightarrow \mathbb{R}$ seminorm where $x=\left(x_{1}, \ldots, x_{m}\right) \in \mathbb{R}^{d^{2}}$,

$$
\|x\|_{T, 1}^{(c)}=\sum_{k=1}^{d} c_{k}\left\|x_{k}\right\|_{T(k)} .
$$

For every $\varepsilon \in(0,1 / 2)$, there exists a data structure for ANN over $\|\cdot\|_{T, 1}^{(c)}$ which achieves approximation $O\left(\frac{\log \log n \cdot \log \log d}{\varepsilon^{4}}\right)$ using space $O\left(d^{2} \cdot n^{1+\varepsilon}\right)$ and query time $O\left(d^{2} \cdot n^{\varepsilon}\right)$.

Proof. The proof follows from Theorem 5.1.2 in [2] and Lemma 5.1.

Finally, we are combine the above results to get an improved algorithm for general symmetric norms.

Theorem 5.3. For every d-dimensional symmetric norm $\|\cdot\|_{X}$ and every $\varepsilon \in(0,1 / 2)$, there exists a data structure for ANN over $\|\cdot\|_{X}$ which achieves approximation $O\left(\frac{\log ^{2} \log n \log \log d}{\varepsilon^{5}}\right)$ using space $d^{O(1)} \cdot O\left(n^{1+\varepsilon}\right)$ and query time $d^{O(1)} \cdot O\left(n^{\varepsilon}\right)$.

Proof. Given Theorem 4.2, we embed $\|\cdot\|_{X}$ into $\bigoplus_{\ell_{\infty}} \bigoplus_{\ell_{1}} T^{(c)}$ with approximation $\left(1 \pm \frac{1}{10}\right)$. The result from Lemma 5.2 allows we to apply Theorem 2.6 to obtain the desired data structure.

Theorem 5.3 implies our main result Theorem 1.1 stated in the introduction.

\section{LOWER BOUNDS}

In this section, we show that our techniques do not extend to general norms. In particular, we show there does not exist a universal norm $U$ for which any norm embeds (possibly randomized) with constant distortion, unless the blow-up in dimension is exponential. Hence the result from below applies to cases of $U=\ell_{\infty}$ as well as an (low-dimensional) product spaces.

Theorem 6.1. For any $\varepsilon>0$, let $U$ be a $d^{\prime}$-dimensional normed space such that for any $d$-dimensional normed space $X$, there exists a 
distribution $\mathcal{D}$ supported on linear embeddings $f: \mathbb{R}^{d} \rightarrow \mathbb{R}^{d^{\prime}}$ where for every $x \in \mathbb{R}^{d}$,

$$
\|x\|_{X} \leq\|f(x)\|_{U} \leq D\|x\|_{U}
$$

holds with probability at least $\frac{2}{3}$ over the draw of $f \sim \mathcal{D}$, for $D=$ $O\left(d^{1 / 2-\varepsilon}\right)$. Then $d^{\prime}=\exp \left(\Omega\left(d^{2 \varepsilon}\right)\right)$.

We will prove the above theorem by showing that if there exists a universal normed space $U$ satisfying the conditions of Theorem 6.1 above, then two parties, call them Alice and Bob, can use the embeddings to solve the communication problem INDEx with only a few bits. Let $U$ be a proposed $d^{\prime}$-dimensional normed space satisfying the conditions of Theorem 6.1. By the John's theorem [13], we may apply a linear transform so that:

$$
B_{\ell_{2}} \subset B_{U} \subset \sqrt{d^{\prime}} B_{\ell_{2}}
$$

Lemma 6.2. For any $\varepsilon>0$, there exists a set of $\exp \left(\Omega\left(d^{2 \varepsilon}\right)\right)$ many points on the unit sphere $S^{d-1}$ such that pairwise inner-products are at most $\frac{1}{d^{1 / 2-\varepsilon}}$. In fact, these points may consist of points whose coordinates are $\pm \frac{1}{\sqrt{d}}$.

Proof. Consider picking two random points $x, y \in S^{d-1}$ where each entry is $\pm \frac{1}{\sqrt{d}}$. Then by Bernstein's inequality,

$$
\operatorname{Pr}_{x, y}\left[|\langle x, y\rangle| \geq \frac{1}{d^{1 / 2-\varepsilon}}\right] \leq 2 \exp \left(-\Omega\left(d^{2 \varepsilon}\right)\right)
$$

We may pick $\exp \left(\Omega\left(d^{2 \varepsilon}\right)\right)$ random points and union bound over the probability that some pair has large inner product.

Fix $\varepsilon>0$ and $C=d^{1 / 2-\varepsilon}$, and let $P$ be set a set of unit vectors with pairwise inner-product at most $\frac{1}{C}$ of size $\exp \left(\Omega\left(d^{2 \varepsilon}\right)\right)$. For each $a \in\{0,1\}^{P}$ consider the following norm:

$$
\|x\|_{a}=C \cdot \max _{y \in P: a_{y}=1}|\langle x, y\rangle| .
$$

Assume there exists a randomized linear embedding $f: \mathbb{R}^{d} \rightarrow \mathbb{R}^{d^{\prime}}$ with the following guarantees:

- For every $x \in \mathbb{R}^{d},\|x\|_{a} \leq\|f(x)\|_{U} \leq D\|x\|_{a}$ with probability at least $\frac{2}{3}$.

Note the embedding $f$ can be described by $M$, a $d^{\prime} \times d$ matrix of real numbers. Additionally, we consider rounding each entry of $M$ by to the nearest integer multiple of $\frac{1}{\operatorname{poly}(d)}$ to obtain $M^{\prime}$. For each $x \in S^{d-1},\left\|\left(M-M^{\prime}\right) x\right\|_{U} \leq\left\|\left(M-M^{\prime}\right) x\right\|_{2} \leq \frac{1}{\operatorname{poly}(d)}$. Thus, we may assume each entry of $M$ is an integer multiple of $\frac{1}{\operatorname{poly}(d)}$, and lose $\left(1 \pm \frac{1}{\operatorname{poly}(d)}\right)$ factor in the distortion of the embedding for vectors in $B_{2}$.

We now show that the existence of the randomized embedding implies a one-way randomized protocol for the communication problem INDEX. We first describe the problem. In an instance of INDEX:

- Alice receives a string $a \in\{0,1\}^{n}$.

- Bob receives an index $i \in[n]$.

- Alice communicates with Bob so that he can output $a_{i}$.

THEOREM 6.3 ([32]). The randomized one-way communication complexity of INDEX is $\Omega(n)$.
We give a protocol for INDEX:

(1) Suppose Alice has input $a \in\{0,1\}^{P}$. She will generate the norm $\|\cdot\|_{a}$ described above. Note that $f \sim \mathcal{D}$ has that for each $x \in \mathbb{R}^{d}$, the embedding preserves the norm of $x$ up to $D$ with probability $\frac{2}{3}$. In particular, if Bob's input is $i \in|P|$, corresponding to point $y$, then an embedding $f \in \mathcal{D}$, which we represent as a $d^{\prime} \times d$ matrix $M$, satisfies:

$$
\|y\|_{a} \leq\|M y\|_{U} \leq D\|y\|_{a}
$$

with probability $\frac{2}{3}$. In particular, with probability $\frac{2}{3}$ :

- If $a_{i}=0$, then $\|y\|_{a} \leq 1$, which implies $\|M y\|_{U} \leq D$.

- If $a_{i}=1$, then $\|y\|_{a} \geq C$, which implies $\|M y\|_{U} \geq C$. Alice computes the set $P_{c} \subset P$ of vectors which satisfy the above property (i.e. the embedding $M$ preserves increases the norm by at most a factor $D$ ).

(2) Alice finds a subset $B \subset P_{c}$ of linearly independent vectors such that every $x \in P_{c}$ we have $x \in \operatorname{span}(B)$. Note that $|B| \leq d$ and for all $x \in B,\|M x\|_{2} \leq \sqrt{d^{\prime}}\|M x\|_{U} \leq C \cdot D$. $\sqrt{d^{\prime}}$. Therefore, each $M x \in \mathbb{R}^{d^{\prime}}$ can be written with $\tilde{O}\left(d^{\prime}\right)$ bits. So Alice sends the set $B$, as well as $M x$ for each $x \in B$ using $\tilde{O}\left(d d^{\prime}\right)$ bits.

(3) In order for Bob to decode $a_{i}$, he first checks whether $y \in \operatorname{span}(B)$, and if not, he guesses. If $y \in \operatorname{span}(B)$, which happens with probability $\frac{2}{3}$, then Bob writes

$$
y=\sum_{b_{i} \in B} c_{i} b_{i}
$$

and $M y=\sum_{b_{i} \in B} c_{i} M b_{i}$. If $\|M y\|_{U} \leq D$, then $a_{i}=0$ and if $\|M y\|_{U} \geq C$ then $a_{i}=1$. Thus, if $D<\frac{C}{2}$, Bob can recover $a_{i}$ with probability $\frac{2}{3}$.

Alice communicates $\tilde{O}\left(d d^{\prime}\right)$ bits, and Bob is able to recover $a_{i}$ with probability $\frac{2}{3}$. By Theorem $6.3, d d^{\prime} \geq \tilde{\Omega}(|P|)$, which in turn implies $d^{\prime} \geq \exp \left(\Omega\left(d^{2 \varepsilon}\right)\right)$.

\section{A BOUNDING SPACE IN ANN FOR PRODUCT SPACES}

Here we justify the space bound of the algorithm from Theorem 2.6 (from [24]). We note that the improved bound was also claimed in [6], albeit without a proof.

First of all, as suggested at the end of Section 3 of [24], one modifies the algorithm to obtain space of the form of $n^{1+\varepsilon}$, at the expense of increasing the approximation to $O\left(\varepsilon^{-1} \log \log n\right)$. This is done by replacing the conditions in Case 2 and 3 by respectively:

$$
\left[\frac{\left|B(s, R(s)+c+1) \cap S_{\mid i}\right|}{\left|S_{\mid i}\right|}\right]^{1+\varepsilon}<\frac{\left|B(s, R(s)) \cap S_{\mid i}\right|}{\left|S_{\mid i}\right|},
$$

and

$$
\left[\frac{\left|S_{\mid i}-B\left(p, R^{\prime}\right)\right|}{|S|}\right]^{1+\varepsilon}<\frac{\left|S_{\mid i}-B\left(s, R^{\prime}+2\right)\right|}{|S|} .
$$

With the remaining algorithm being precisely the same, our only task here is to argue the space bound. First of all we bound the sum of the number of points stored in all the leaves. For a tree with $m$ nodes, let $L(m)$ be an upper bound on this count. We would like to prove that $L(m) \leq m^{1+\varepsilon}$. As in [24], we only need to focus on cases 2 and 3 of the construction, as case 1 does not replicate the points. We will consider the case 2 (case 3 is exactly similar). 
Let $m_{j}=\left|S_{j}\right|$ and $m_{j}^{\prime}=\left|S_{\mid i} \cap \cup_{s \in S_{j}} B(s, c+1)\right|$, whereas $|S|=m$. By construction, we have that $\sum m_{j}=m$ and $m_{j} / m>\left(m_{j}^{\prime} / m\right)^{1+\varepsilon}$ for all $j$.

By induction, assume $L\left(m_{j}^{\prime}\right) \leq\left(m_{j}^{\prime}\right)^{1+\varepsilon}$ for all children. Then, we have that:

$$
L(m) \leq \sum_{j} L\left(m_{j}^{\prime}\right) \leq \sum_{j}\left(m_{j}^{\prime}\right)^{1+\varepsilon}<m^{\varepsilon} \sum_{j} m_{j}=m^{1+\varepsilon} .
$$

We now argue the total space is $O\left(S(n) \cdot k \log n \cdot n^{\epsilon}\right)$. Since the depth of the tree is $O(k \log n)$, we have that the total number of points stored in the ANN data structures is $O(k \log n \cdot C(n))=$ $O\left(k \log n \cdot n^{1+\epsilon}\right)$. Since each ANN is on at most $n$ points, we have that, for each occurrence of a point in the ANN data structure, we have an additional factor of $S(n) / n .{ }^{4}$ Hence the total space occupied by all the ANN data structures is $O\left(S(n) / n \cdot k \log n \cdot n^{1+\epsilon}\right)$. Using a smaller $\varepsilon$ (to hide the $\log n$ factor), we obtain the stated space bound of $O\left(S(n) \cdot k \cdot n^{\epsilon}\right)$.

\section{B $\widetilde{O}($ LOG $D$ )-ANN FOR SYMMETRIC NORMS}

We provide a simple ANN algorithm for general symmetric norm achieving $O(\log d \log \log d)$ approximation using near-linear space and sub-linear query time. The algorithm will leverage the results in the previous section by relating general symmetric norms to Orlicz norms. Recall the definition of level vectors in Definition 4.12.

Definition B.1. Let $\|\cdot\|_{X}$ be any symmetric norm. Let $L_{k}>0$ be the minimum number of coordinates needed at level $k$ to have norm at least 1 . In other words,

$$
L_{k}=\min \left\{j \in[d] \mid\left\|\beta^{-i} \xi^{(j)}\right\|_{X}>1\right\} .
$$

At a high level, we will relate the norm of a vector $x \in \mathbb{R}^{d}$ to the norm of its level vectors $V_{k}(x)$. The definition above gives a way to measure the contribution of level $k$ to the norm. For example, if $x \in \mathbb{R}^{d}$ has norm $\|x\|_{X} \geq D$, and there are only $2 \log _{\beta} d$ non-zero levels with respect to $x$, then some level vector $\left\|V_{k}(x)\right\|_{X} \geq \frac{D}{2 \log _{\beta} d}$. This implies $b_{k}=\Omega\left(\frac{D L_{k}}{\log _{\beta} d}\right)$, since we may divide $V_{k}(x)$ into a sum of vectors with $L_{k}$ coordinates at level $k$.

On the other hand, if $x \in \mathbb{R}^{d}$ has $\|x\|_{X} \leq 1$, then $b_{k}<L_{k}$ for each $k$. Since we consider only $2 \log _{\beta} d$ relevant levels, for $\|x\|_{S} \leq 1$,

$$
\sum_{k=0}^{2 \log _{\beta} d-1} \frac{b_{k}}{L_{k}} \leq 2 \log _{\beta} d .
$$

Additionally, $\sum_{k=0}^{2 \log _{\beta} d-1}\left(b_{k} / L_{k}\right)$ can be decomposed as an additive contribution of coordinates. In particular, coordinate $x_{i}$ contributes $1 / L_{k}$ if $i \in B_{k}$. Therefore, we can hope to approximate the symmetric norm by an Orlicz norms and apply the arguments from Lemma 3.1.

The lemma below formalizes the ideas discussed above.

Lemma B.2. Let $\|\cdot\|_{X}$ be any symmetric norm. For any $D, \alpha>1$, there exists a non-decreasing function $G: \mathbb{R}_{+} \rightarrow \mathbb{R}_{+}$with $G(0)=0$ and $G(t) \rightarrow \infty$ as $t \rightarrow \infty$, where every vector $x \in \mathbb{R}^{d}$ satisfies the following:

${ }^{4}$ Here we assume the natural condition that $S(n)$ is increasing, which is, otherwise, easy to guarantee.
- If $\|x\|_{X} \leq 1$, then $\sum_{i=1}^{d} G\left(\left|x_{i}\right|\right) \leq 2 \log _{\beta} d$.

- If $\|x\|_{X}>\alpha D \cdot 7 \log _{\beta} d$, then $\sum_{i=1}^{d} G\left(\frac{\left|x_{i}\right|}{D}\right) \geq \alpha \cdot 2 \log _{\beta} d$.

Proof. For $i \geq 0$, let $A_{i}=\left(\beta^{-i-1}, \beta^{-i}\right]$. The function $G: \mathbb{R}_{+} \rightarrow$ $\mathbb{R}_{+}$is defined as

$$
G(t)=\sum_{i=0}^{2 \log _{\beta} d-1} \frac{\chi_{A_{i}}(t)}{L_{i}}+\alpha \cdot 2 \log _{\beta} d \cdot t \cdot \chi_{(1, \infty)}(t)
$$

Note that $G(0)=0$ and $G(t) \rightarrow \infty$ as $t \rightarrow \infty$.

Recall the norm satisfies, $\left\|\xi^{(1)}\right\|_{X}=1$, so if $\|x\|_{X} \leq 1$, then $\left|x_{i}\right| \leq 1$ for all $i \in[d]$. This means $\chi_{(1, \infty)}\left(\left|x_{i}\right|\right)=0$ so the second term of the RHS of (2) is zero. Therefore,

$$
\sum_{i=1}^{d} G\left(\left|x_{i}\right|\right)=\sum_{i=1}^{d} \sum_{k=0}^{2 \log _{\beta} d-1} \frac{\chi_{A_{k}}\left(\left|x_{i}\right|\right)}{L_{k}}=\sum_{k=0}^{2 \log _{\beta} d-1} \frac{b_{k}}{L_{k}}
$$

where $b_{k}$ is defined with respect to $x$. Since, $b_{k}<L_{k}$ for all $0 \leq$ $k<2 \log _{\beta} d$,

$$
\sum_{i=1}^{d} G\left(\left|x_{i}\right|\right) \leq 2 \log _{\beta} d
$$

If $x \in \mathbb{R}^{d}$ where $\|x\|_{X}>\alpha D \cdot 7 \log _{\beta} d$, then the vector $\left\|\frac{x}{D}\right\|_{X}>$ $\alpha \cdot 7 \log _{\beta} d$. So it suffices to prove that for any vector $x \in \mathbb{R}^{d}$ with $\|x\|_{X}>\alpha \cdot 7 \log _{\beta} d$,

$$
\sum_{i=1}^{d} G\left(\left|x_{i}\right|\right) \geq \alpha \cdot 2 \log _{\beta} d
$$

Additionally, for any vector $x \in \mathbb{R}^{d}$, we may consider the vector $C(x) \in \mathbb{R}^{d}$ for $\tau=\frac{\beta}{d^{2}}$ from Definition 4.13. By Proposition 4.15, $\|C(x)\|_{X} \geq\|x\|_{X}-\frac{\beta}{d}>\alpha \cdot 6 \log _{\beta} d$. Therefore, we may assume $x \in \mathbb{R}^{d}$ has $\|x\|_{X}>\alpha \cdot 6 \log _{\beta} d$, and that all non-zero coordinates have absolute values greater than $\frac{\beta}{d^{2}}$. Equivalently, $b_{k}=0$ for all $k \geq 2 \log _{\beta} d$. If for some $i \in[d],\left|x_{i}\right| \geq 1$, then the second term in the RHS of (2) is non-zero, and $G\left(\left|x_{i}\right|\right) \geq \alpha \cdot 2 \log _{\beta} d$. So we may further assume all coordinates of $x$ lie in levels $k=0, \ldots, 2 \log _{\beta} d-1$. Note that

$$
\sum_{i=1}^{d} G\left(\left|x_{i}\right|\right)=\sum_{k=0}^{2 \log _{\beta} d-1} \sum_{i=1}^{d} G\left(\left|V_{k}(x)_{i}\right|\right)
$$

and for each $0 \leq k<2 \log _{\beta} d, \sum_{i=1}^{d} G\left(\left|V_{k}(x)_{i}\right|\right)=\frac{b_{k}}{L_{k}}$.

We partition the levels into two groups,

$$
A=\left\{k \mid \frac{b_{k}}{L_{k}}<1\right\} \quad \text { and } \quad B=\left\{k \mid \frac{b_{k}}{L_{k}} \geq 1\right\} .
$$

For all $k \in B$,

$$
\left\|V_{k}(x)\right\|_{X} \leq\left\lceil\frac{b_{k}}{L_{k}}\right\rceil \leq \frac{2 b_{k}}{L_{k}}
$$

since by the triangle inequality, we can break $V_{k}(x)$ into at most $\left\lceil\frac{b_{k}}{L_{k}}\right\rceil$ vectors with $L_{k}$ coordinates at level $k$ each having norm at least 1. 

Then

Suppose for the sake of contradiction that $\sum_{k \in B} \frac{b_{k}}{L_{k}} \leq \alpha \cdot 2 \log _{\beta} d$.

$$
\alpha \cdot 4 \log _{\beta} d \geq \sum_{k \in B} \frac{2 b_{k}}{L_{k}} \geq \sum_{k \in B}\left\|V_{k}(x)\right\|_{X} .
$$

Additionally, since $\|x\|_{X}>\alpha \cdot 6 \log _{\beta} d$, and

$$
\alpha \cdot 6 \log _{\beta} d<\|x\|_{X} \leq \sum_{k \in A}\left\|V_{k}(x)\right\|_{X}+\sum_{k \in B}\left\|V_{k}(x)\right\|_{X},
$$

it follows that

$$
\sum_{k \in A}\left\|V_{k}(x)\right\|_{X}>\alpha \cdot 2 \log _{\beta} d .
$$

However, this is a contradiction for since $|A| \leq 2 \log _{\beta} d$ and $\left\|V_{k}(x)\right\|_{X} \leq 1$.

Lemma B.3. For any $\varepsilon \in(0,1 / 2)$, there exists a data structure for ANN over any symmetric norm $\|\cdot\|_{X}$ which achieves approximation $O\left(\frac{\log d \log \log d}{\varepsilon^{2}}\right)$ using space $O\left(d n^{1+\varepsilon}\right)$ and query time $O\left(d n^{\varepsilon}\right)$.

Proof. We fix $\beta=\frac{3}{2}$. The proof of this lemma follows in the same way as the proof of Theorem 3.2. The one difference is that we rescale the $\ell_{\infty}$ norm by $\frac{1}{2 \log _{\beta} d}$ after applying the embedding.

\section{B.1 The $\log ^{\Omega(1)} d$-approximation is Necessary}

Let us remark that we cannot push the technique much further. Namely, any $G(\cdot)$ (even non-convex) requires approximation $\Omega(\sqrt{\log d})$ for the following norm. Define the norm of a vector to be

$$
\|x\|=\max _{1 \leq k \leq d}\left(\frac{x_{1}^{*}+x_{2}^{*}+\ldots x_{k}^{*}}{\sqrt{k}}\right) .
$$

This is the minimal norm for $a_{k}=\sqrt{k}$ (see Section 1.2 for the definition). It is not hard to check that an approximation with any $G(\cdot)$ ends up having a distortion $\Omega(\sqrt{\log d})$.

The idea is to consider the following vectors: for every $1 \leq k \leq d$, we consider a vector

$$
(\underbrace{1,1, \ldots, 1}_{k}, 0,0, \ldots, 0),
$$

and besides, we consider a vector

$$
(1, \sqrt{2}-1, \sqrt{3}-\sqrt{2}, \ldots, \sqrt{d}-\sqrt{d-1}) .
$$

The remaining calculation is a simple exercise.

\section{LOWER BOUND FOR ARBITRARY METRICS: EXPANDER GRAPHS}

We give an example of a metric that is hard for current approaches to ANN search. The lower bound is based on the notion of robust expansion, which implies all known lower bounds for ANN $[9,39]$. In what follows, we will refer to $d=\log N$ as the dimension of a finite metric space of size $N$.

Our example of a hard metric will be the shortest path metric on any spectral expander graph. We note that a similar theorem to the one below is also known for a finite subset of the high-dimensional Earth-Mover Distance [28].

Fix $M$ to be the metric induced by the shortest path distance on a 3-regular expander $G$ on $N$ nodes. In particular, assume that
$1-\lambda(G)>c$, where $c$ is an absolute constant, and $\lambda(G) \in(0,1)$ is the second-largest eigenvalue of the normalized adjacency matrix of $G$. Let $d$ be the dimension $d=\log N$.

Theorem C.1. For any approximation $\alpha>1$, and data set size $n \geq 1$ with $d^{\Omega(1)} \leq n \leq N^{O(1)}$, any $\alpha-A N N$ data structure on $n$ points which makes $t$ cell probes (with cells of size at most $\left.w \leq(d \log n)^{O(1)}\right)$, and has success probability at least $\gamma>n^{-1+o(1)}$, must use space $m=\gamma^{\Omega(1 / t)} N^{\Omega(1 /(\alpha t))}=\gamma^{\Omega(1 / t)} 2^{\Omega(d /(\alpha t))}$.

We proceed by introducing a few definitions from [39], and then prove lower bounds on the robust expansion.

Definition C.2 ([39]). In the Graphical Neighbor Search problem (GNS), we are given a bipartite graph $H=(U, V, E)$ where the dataset comes from $U$ and the queries come from $V$. The dataset consists of pairs $P=\left\{\left(p_{i}, x_{i}\right) \mid p_{i} \in U, x_{i} \in\{0,1\}, i \in[n]\right\}$. On query $q \in V$, if there exists a unique $p_{i}$ with $\left(p_{i}, q\right) \in E$, then we want to return $x_{i}$

One can use the GNS problem to prove lower bounds on $c$-ANN as follows: build a GNS graph $H$ by taking $U=V=[N]$, and connecting two points $u \in U, v \in V$ iff they are at a distance at most $r$ (see details in [39]). We will also need to make sure that in our instances $q$ is not closer than $r r$ to other points except the near neighbor.

We now introduce the notion of robust expansion, used in [39] to prove lower bounds.

Definition C.3 (Robust Expansion [39]). For a GNS graph $H=$ $(U, V, E)$, fix a distribution $e$ on $E \subset U \times V$, and let $\mu$ be the marginal on $U$ and $\eta$ be the marginal on $V$. For $\delta, \gamma \in(0,1]$, the robust expansion $\Phi_{r}(\delta, \gamma)$ is defined as follows:

$$
\Phi_{r}(\delta, \gamma)=\min _{A \subset V: \eta(A) \leq \delta} \min _{B \subset U: \frac{e(A \times B)}{e(A \times V)} \geq \gamma} \frac{\mu(B)}{\eta(A)} .
$$

We now prove a lower bound on the robust expansion $\Phi_{r}(\delta, \gamma)$ for a GNS graph arising from the shortest path metric on the expander graph $G$. Fix $r=d / \alpha$. The hard distribution $e$ is defined as follows: pick $p$ at random from $M$ and obtain $q$ by running a random walk of length $r$ starting at $p$. Note that for $n<N^{1 / 4}$ and sufficiently high constant $\alpha$, the distribution satisfies the weak-independence condition required for applying the results in [39].

Fix any sets $A, B \subset M$, where $a=|A| / N$ and $b=|B| / N$. By the expander mixing lemma applied to $G^{r}$, we obtain that:

$$
\left|E_{G^{r}}(A, B)-\frac{|A| \cdot|B|}{3^{r} N}\right| \leq \lambda^{3} 3^{r} \sqrt{|A| \cdot|B|}
$$

Considering that $\operatorname{Pr}[q \in B \mid p \in A]=\frac{E_{G} r(A, B)}{a N \cdot 3^{r}}$, we have that:

$$
\operatorname{Pr}[q \in B \mid p \in A] \leq b+\lambda^{r} \sqrt{b / a} .
$$

Restricting to sets $A, B$ such that $\operatorname{Pr}[q \in B \mid p \in A] \geq \gamma$, for which we must have that $\Phi_{r}=\Phi_{r}(a, \gamma) \geq b / a$ (by definition), we conclude:

$$
\begin{gathered}
\gamma \leq \Phi_{r} \cdot a+\lambda^{r} \sqrt{\Phi_{r}} . \\
\text { Hence, either } \Phi_{r}=\Omega(\gamma / a) \text { or } \Phi_{r}=\Omega\left(\gamma^{2} / \lambda^{2 r}\right) .
\end{gathered}
$$

Proof of Theorem C.1. Applying Theorem 1.5 from [39], we have that, for $t \geq 1$ cell probes, either: 1) $m^{t} w / n \geq \Omega\left(\gamma \cdot m^{t}\right)$, an impossibility; or, 2) $m^{t} w / n \geq \Omega\left(\gamma^{2} / \lambda^{2 r}\right)$, or $m^{t}=\Omega\left(\frac{n}{w} \gamma^{2} / \lambda^{2 r}\right)$, implying $m=\gamma^{2 / t} N^{\Omega(1 /(\alpha t))}$. 
To show how bad the situation for expander metrics is, we state a lower bound on for $\alpha$-ANN on the expander metric described above in the list-of-points model, which captures the hashing-based algorithms of [9] and in the decision tree model of [23]. The proofs follow from a simple derivation using the robust expansion lower bounds in Section 7 of [9] and a reduction of decision trees to $O(\log m)$-cell-probe data structures similar to Appendix A in [4].

Theorem C.4. Any list-of-points data structure for (c, $r)$-ANN for random instances of $n$ points in the expander metric of dimensiond (described above) with query time $t$ and space $m$ has either $t=\Omega(n)$, or $m=\exp (\Omega(d))$.

Theorem C.5. Let $d=\Omega\left(\log ^{1+\varepsilon} n\right)$ for some $\varepsilon>0$. Any decision tree of size $m$ and depth $t$ and word size $w$ succeeding with probability $\gamma$ satisfies:

$$
\frac{m^{O(\log m)} t w}{n} \geq \Phi_{r}\left(\frac{1}{m^{O(\log m)}}, \frac{\gamma}{O(\log m)}\right) .
$$

In particular, for any $\rho>0$, if $w \leq n^{\rho}$, either $t \geq \widetilde{\Omega}\left(n^{1-\rho}\right)$ or $m=\exp \left(\Omega\left(d^{\varepsilon /(1+\varepsilon)}\right)\right) \operatorname{poly}(n)$.

\section{REFERENCES}

[1] Amirali Abdullah and Suresh Venkatasubramanian. 2015. A Directed Isoperimetric Inequality with application to Bregman Near Neighbor Lower Bounds. In Proceedings of the 47th ACM Symposium on the Theory of Computing (STOC '2015). 509-518.

[2] Alexandr Andoni. 2009. Nearest Neighbor Search: the Old, the New, and the Impossible. Ph.D. Dissertation. MIT.

[3] Alexandr Andoni. 2016. High-Dimensional Computational Geometry. In Hand book of Big Data., Peter Bühlmann, Petros Drineas, Michael Kane, and Mark J van der Laan (Eds.). Chapman and Hall/CRC, 105-123. http://www.crcnetbase com/doi/abs/10.1201/b19567-11

[4] Alexandr Andoni, Dorian Croitoru, and Mihai Patrascu. 2008. Hardness of Nearest Neighbor under L-infinity. In Proceedings of the 49th Annual IEEE Symposium on Foundations of Computer Science (FOCS '2008). 424-433.

[5] Alexandr Andoni and Piotr Indyk. 2006. Near-Optimal Hashing Algorithms for Approximate Nearest Neighbor in High Dimensions. In Proceedings of the 47th Annual IEEE Symposium on Foundations of Computer Science (FOCS '2006). 459-468.

[6] Alexandr Andoni, Piotr Indyk, and Robert Krauthgamer. 2009. Overcoming the $\ell_{1}$ Non-Embeddability Barrier: Algorithms for Product Metrics. In Proceedings of the 20th ACM-SIAM Symposium on Discrete Algorithms (SODA '2009). 865-874.

[7] Alexandr Andoni, Piotr Indyk, Huy L. Nguyen, and Ilya Razenshteyn. 2014 Beyond Locality-Sensitive Hashing. In Proceedings of the 25th ACM-SIAM Symposium on Discrete Algorithms (SODA '2014). 1018-1028. Available as arXiv:1306.1547.

[8] Alexandr Andoni, Robert Krauthgamer, and Ilya Razenshteyn. 2015. Sketching and Embedding are Equivalent for Norms. In Proceedings of the 47th ACM Symposium on the Theory of Computing (STOC '2015). 479-488. Available as arXiv:1411.2577.

[9] Alexandr Andoni, Thijs Laarhoven, Ilya Razenshteyn, and Erik Waingarten. 2017. Optimal Hashing-based Time-Space Trade-offs for Approximate Near Neighbors. In Proceedings of the 28th ACM-SIAM Symposium on Discrete Algorithms (SODA '2017). Available as arXiv:1608.03580.

[10] Alexandr Andoni, Huy L. Nguyen, Aleksandar Nikolov, Ilya Razenshteyn, and Erik Waingarten. 2017. Approximate Near Neighbor for General Symmetric Norms. In Proceedings of the 49th ACM Symposium on the Theory of Computing (STOC '2017). Available at http://arxiv.org/abs/1611.06222.

[11] Alexandr Andoni and Ilya Razenshteyn. 2015. Optimal Data-Dependent Hashing for Approximate Near Neighbors. In Proceedings of the 47th ACM Symposium on the Theory of Computing (STOC '2015). 793-801. Available as arXiv:1501.01062.

[12] Andreas Argyriou, Rina Foygel, and Nathan Srebro. 2012. Sparse Prediction with the $k$-Support Norm. In Proceedings of Advances in Neural Information Processing Systems 25 (NIPS '2012). 944-952.

[13] Keith Ball. 1997. An Elementary Introduction to Modern Convex Geometry. MSRI Publications, Vol. 31. Cambridge University Press.

[14] Yair Bartal and Lee-Ad Gottlieb. 2015. Approximate nearest neighbor search for $\ell_{p}$-spaces $(2<p<\infty)$ via embeddings. (2015). Available as arXiv:1512.01775

[15] Colin Bennett and Robert Sharpley. 1988. Interpolation of Operators. Pure and Applied Mathematics, Vol. 129. Academic Press.
[16] Alina Beygelzimer, Sham Kakade, and John Langford. 2006. Cover trees for nearest neighbor. In Proceedings of the 23rd International Conference on Machine Learning (ICML '2006). 97-104.

[17] Jarosław Błasiok, Vladimir Braverman, Stephen R. Chestnut, Robert Krauthgamer, and Lin F. Yang. 2015. Streaming Symmetric Norms via Measure Concentration. (2015). Available as arXiv:1511.01111.

[18] Vladimir Braverman and Rafail Ostrovsky. 2010. Zero-one frequency laws. In Proceedings of the 42rd ACM Symposium on the Theory of Computing (STOC '2010). 281-290.

[19] Ken Clarkson. 1999. Nearest Neighbor Queries in Metric Spaces. Discrete Comput. Geom. 22, 1 (1999), 63-93. Previously in SoCG97.

[20] Kenneth L. Clarkson and David P. Woodruff. 2015. Sketching for $M$-Estimators: A Unified Approach to Robust Regression. In Proceedings of the 26th ACM-SIAM Symposium on Discrete Algorithms (SODA '2015). 921-939.

[21] S. J. Dilworth and S. J. Montgomery-Smith. 1993. The distribution of vectorvalued Rademacher series. Annals of Probability 21, 4 (1993), 2046-2052.

[22] Sariel Har-Peled, Piotr Indyk, and Rajeev Motwani. 2012. Approximate Nearest Neighbor: Towards Removing the Curse of Dimensionality. Theory of Computing 8, 1 (2012), 321-350.

[23] Piotr Indyk. 2001. On Approximate Nearest Neighbors under $\ell_{\infty}$ Norm. $\mathcal{F}$. Comput. System Sci. 63, 4 (2001), 627-638.

[24] Piotr Indyk. 2002. Approximate nearest neighbor algorithms for Fréchet distance via product metrics. In Proceedings of the 18th ACM Symposium on Computational Geometry (SoCG '2002). 102-106.

[25] Piotr Indyk. 2004. Approximate Nearest Neighbor under edit distance via product metrics. In Proceedings of the 15th ACM-SIAM Symposium on Discrete Algorithms (SODA '2004). 646-650.

[26] Piotr Indyk and Rajeev Motwani. 1998. Approximate Nearest Neighbors: Towards Removing the Curse of Dimensionality. In Proceedings of the 30th ACM Symposium on the Theory of Computing (STOC '1998). 604-613.

[27] Fritz John. 1948. Extremum problems with inequalities as subsidiary conditions. In Studies and Essays Presented to R. Courant on his 60th Birthday, Fanuary 8, 1948. Interscience Publishers, Inc., New York, N. Y., 187-204.

[28] Michael Kapralov and Rina Panigrahy. 2012. NNS Lower Bounds via Metric Expansion for $\ell_{\infty}$ and EMD. In Proceedings of the 39th International Colloquium on Automata, Languages and Programming (ICALP '2012). 545-556.

[29] David R. Karger and Matthias Ruhl. 2002. Finding nearest neighbors in growthrestricted metrics. In Proceedings of the 34th ACM Symposium on the Theory of Computing (STOC '2002). 741-750.

[30] Felix Krahmer and Rachel Ward. 2016. A Unified Framework for Linear Dimensionality Reduction in L1. RIM 70, 1 (2016), 209-231.

[31] Robert Krauthgamer and James R. Lee. 2004. Navigating nets: simple algorithms for proximity search. In Proceedings of the 15th ACM-SIAM Symposium on Discrete Algorithms (SODA '2004). 798-807.

[32] Ilan Kremer, Noam Nisan, and Dana Ron. 1999. On randomized one-round communication complexity. Computational Complexity (1999), 21-49.

[33] Albert W. Marshall, Ingram Olkin, and Barry C. Arnold. 2011. Inequalities: theory of majorization and its applications. Academic press.

[34] Jiři Matoušek. 1996. On the distortion required for embedding finite metric spaces into normed spaces. Israel fournal of Mathematics 93 (1996), 333-344.

[35] Andrew M. McDonald, Massimiliano Pontil, and Dimitris Stamos. 2014. Spectral $k$-Support Norm Regularization. In Proceedings of Advances in Neural Information Processing Systems 25 (NIPS '2014). 3644-3652.

[36] Assaf Naor. 2017. A spectral gap precludes low-dimensional embeddings. In Proceedings of the 33rd International Symposium on Computational Geometry (SoCG '2017).

[37] Assaf Naor and Yuval Rabani. 2006. On approximate nearest neighbor search in $\ell_{p}, p>2$. (2006). Manuscript.

[38] Assaf Naor and Gideon Schechtman. 2007. Planar Earthmover is not in $L_{1}$. SIAM 7. Comput. 37, 3 (2007), 804-826. An extended abstract appeared in FOCS'06.

[39] Rina Panigrahy, Kunal Talwar, and Udi Wieder. 2010. Lower Bounds on Near Neighbor Search via Metric Expansion. In Proceedings of the 51st Annual IEEE Symposium on Foundations of Computer Science (FOCS '2010). 805-814.

[40] Jun Wang, Wei Liu, Sanjiv Kumar, and Shih-Fu Chang. 2015. Learning to Hash for Indexing Big Data - A Survey. (2015). Available as arXiv:1509.05472.

[41] Jingdong Wang, Heng Tao Shen, Kingkuan Song, and Jianqiu Ji. 2014. Hashing for Similarity Search: a Survey. (2014). Available as arXiv:1408.2927.

[42] Przemysław Wojtaszczyk. 1991. Banach Spaces for Analysts. Cambridge University Press. 